\title{
Effect ofX-ray Irradiation on Structural and Optical Properties of Topological Insulator Bismuth Telluride Nano-Structure Thin Film
}

\author{
Ahmed A. Khodiri, Ahmed M. Nawar, K.M Abd El-kader \\ Department of Physics, Faculty of Science, Suez Canal University, Ismailia, Egypt
}

\begin{abstract}
Bismuth Telluride $\left(\mathrm{Bi}_{2} \mathrm{Te}_{3}\right)$ thin films were grown ontooptical flat fused quartz and chronic glass by using a thermal evaporation technique for optical and structural investigations, respectively. The prepared films were divided into three groups; the first group was as-grown films, and the second and third groups were irradiated with $\mathrm{X}$-ray radiation with energies 6 and $15 \mathrm{MeV}$.The crystal structure and morphology of the grown $\mathrm{Bi}_{2} \mathrm{Te}_{3}$ thin films were identified by X-ray diffraction (XRD) and scan electron microscopy (SEM) before and after exposed to $X$-ray irradiation. The optical constants(Refractive index, $n$, and absorption index, $k$ ) of asgrown and irradiated $\mathrm{Bi}_{2} \mathrm{Te}_{3}$ thin films were estimated and calculated in the wavelength range from 200 to 2500 $\mathrm{nm}$ by using spectrophotometric measurements of transmittance and reflectance at normal incidence. The estimated onset optical gap $E_{g}$ for as-grown equal to $0.35 \mathrm{eV}$ and this value was found to be decreased corresponding to the increasing in X-ray radiation energy.The variation of the refractive index of normal dispersion is well described by the single oscillator model. The dielectric constant at infinite frequency $\left(\varepsilon_{\infty}\right)$, the lattice dielectric constant $\left(\varepsilon_{L}\right)$ and the ratio of free carrier concentration of the effective mass $(\mathrm{N} / \mathrm{m} *)$ are calculated. Finally, the nonlinear optical parameters are calculated using some empirical relations.
\end{abstract}

Keywords:bismuth telluride; thin film; X-ray irradiation; the linear dispersionparameters; the non-linear dispersion parameters

\section{Introduction}

Bismuth-based tritellurides and triselenides, which are well-known thermoelectric materials because they possess the highest thermoelectric figure of merit known at room temperature [1] It have been used in thermoelectric refrigerators for the temperature control of semiconductor devices such as laser diodes or CCDs (charge coupled devices)[2], optical recording systems [3],magneto-resistance [4], and strain gauges [5].Recently, $\mathrm{Bi}_{2} \mathrm{Te}_{3}$ was predicted to be three-dimensional (3D) topological insulator (TI); a new class of quantum matter with conductive massless Dirac fermions on the surface [6]. The 3D-TIs possess fully gapped bulk states and gapless surface states, which can be described by the Rashba spin-orbit Hamiltonian [79].Bismuth Telluride thin films have been fabricated by using different techniques such as; flash thermal evaporation, sputtering, electrochemical deposition, metal-organic chemical vapor deposition and mechanically exfoliated method [10-17].In this work, the authors aim to fabricate $\mathrm{Bi}_{2} \mathrm{Te}_{3}$ thin films by thermal evaporation techniqueat substrate temperature $623 \mathrm{~K}$ and investigate the effects of $\mathrm{x}$-ray irradiation process on the structural and optical constants (Refractive index, $\mathrm{n}$, and absorption index, $\mathrm{k}$ ) and nonlinear optical properties.

The interaction of ionizing radiations (such as $\mathrm{X}$-rays, gamma rays, etc.) with material, mainly, occurs by means of electronic excitation, electronic ionization, and, primarily, atomic displacement of the orbital electrons [18]. The influence of radiation on the material depends on dose rate and the parameters of the films. The degradation is more severe for the higher dose and the thinner films $[19,20] \cdot \mathrm{Bi}_{2} \mathrm{Te}_{3}$ thin films have been studied But much of the optical properties of $\mathrm{Bi}_{2} \mathrm{Te}_{3}$ are unknown. The exposure of solid to ionizing radiations produces changes in the microstructural properties of the material, which in turn affects the optical properties. study of these changes is quite important, not only to understand physicochemical functions and spectroscopic properties of this material but also to increase their applicability in different fields and enable information about the induced irradiation defects and their interaction with the matter components. The main purpose of this work is study in detail the induced changes in the topological and the morphological nanostructures of $\mathrm{Bi}_{2} \mathrm{Te}_{3}$ thin films and estimate the related optical and dispersion parameters of these films before and after exposed to X-ray radiation.

\section{Experimental procedures}

Bismuth Telluride $\mathrm{Bi}_{2} \mathrm{Te}_{3}$ in powder form with a purity of $99.99 \%$ was purchased from Aldrich Chem. $\mathrm{Bi}_{2} \mathrm{Te}_{3}$ thin films were grown by a vacuum thermal evaporation technique using a high vacuum coating unit (Edward, E $306 \mathrm{~A}$, England), under a pressure of about $4 \times 10^{-4} \mathrm{~Pa}$. Which deposited on a flat glass substrates for the structural properties and quartz substrates for the optical properties. A boat-shaped tungsten filament was 
used to thermally evaporate $\mathrm{Bi}_{2} \mathrm{Te}_{3}$ in a vacuum.The films are divided into two groups. The first (as-grown) group is characterized as prepared. The second group was irradiated in air, at temperature $299.3 \mathrm{~K}$ and airpressure $1010.1 \mathrm{hPawith}$ field size $10 \times 10 \mathrm{~cm}$ at SSD $100 \mathrm{~cm}$ by high energy X-ray (6 MeV and $15 \mathrm{MeV}$ ) with a dose rate $4 \mathrm{~Gy} / \mathrm{min}$ using aClinac (Dual energy Linear Accelerator DMX - Varian). The exposure time for irradiation process is constant $2.5 \mathrm{~min}$ and the exposed dose for $6 \mathrm{MeV}$ is $10 \mathrm{~Gy} \pm 0.018$ and for $15 \mathrm{MeV} 10$ Gy \pm 0.01 ; the output X-ray radiation beam calibrated using (UNIDOS E T10008-80685 Electrometer, TM30010-03870 Ionization Chamber, PTW Freiburg, Germany). The optical absorption spectra for the $\mathrm{Bi}_{2} \mathrm{Te}_{3}$ thin films of thickness $36 \mathrm{~nm}$, exposed to different levels of the X-ray radiation energy, were recorded using (JASCO model V-570 UV-VIS-NIR) Spectrometer for the wavelengths in the range 200-2500 $\mathrm{nm}$. These absorption spectra were analyzed to obtain the dose dependence of the optical band gap. The SEM images, for the $\mathrm{Bi}_{2} \mathrm{Te}_{3}$ thin films have been analyzed using $\mathrm{X}$ - ray Diffraction (model $\mathrm{X}^{\prime}$ pert) with utilized monochromatic $\mathrm{Cu}-\mathrm{K} \alpha$ radiation $(\lambda=1.54056 \AA)$. In the degrees range of $10^{\circ}-65^{\circ}$. In order to understand the changes in the structural and optical properties due to X-ray irradiation.

In order to calculate the optical constants, the absorption coefficient, $\alpha$, the absorption index, $\mathrm{k}$, and the refractive index, $\mathrm{n}$, of the films at different wavelengths, we canuse the following equations [21,22]:

$$
\begin{aligned}
& \alpha=\frac{1}{d} \ln +\left[\frac{(1-R)^{2}}{2 T}+\left\{\frac{(1-R)^{4}}{4 T^{2}}-R^{2}\right\}^{1 / 2}\right] \\
& \mathbf{k}=\square \square / 4(1) \\
& n=\frac{1+R}{1-R}+\sqrt{\frac{4 R}{(1-R)^{2}}-K^{2}}(3)
\end{aligned}
$$

Where $\alpha$ is the absorption coefficient and $\mathrm{d}$ is the film thickness.

\subsection{X-ray diffraction analysis}

\section{Results and discussion}

Figure 1 show the spectrum of X-ray powder diffraction for $\mathrm{Bi}_{2} \mathrm{Te}_{3}$ in powder form were taken in a $2 \theta$ range from $10^{\circ}$ to $65^{\circ}$. The pattern has many diffraction peaks with different intensities indicating that the powder of $\mathrm{Bi}_{2} \mathrm{Te}_{3}$ has a polycrystalline nature. The highest preferred orientation is found along the (0 1 15$)$ plane. The unit cell parameters of $\mathrm{Bi}_{2} \mathrm{Te}_{3}$ in powder form were determined and the parameters found to be $\mathrm{a}=4.381 \AA$ and $\mathrm{c}=30.437 \AA \hat{\text {. }}$. All the diffraction peaks in the patterns of all the powder products correspond to the peaks of rhombohedral Bi2Te3 (JCPDS no. 08-0021) with space group: R3m (166). The cell parameters in JCPDs no. 08$0021, \mathrm{a}=4 \cdot 384 \AA$ and $\mathrm{c}=30 \cdot 45 \AA$. Therefore, the obtained powder product is rhombohedral $\mathrm{Bi}_{2} \mathrm{Te}_{3}$.

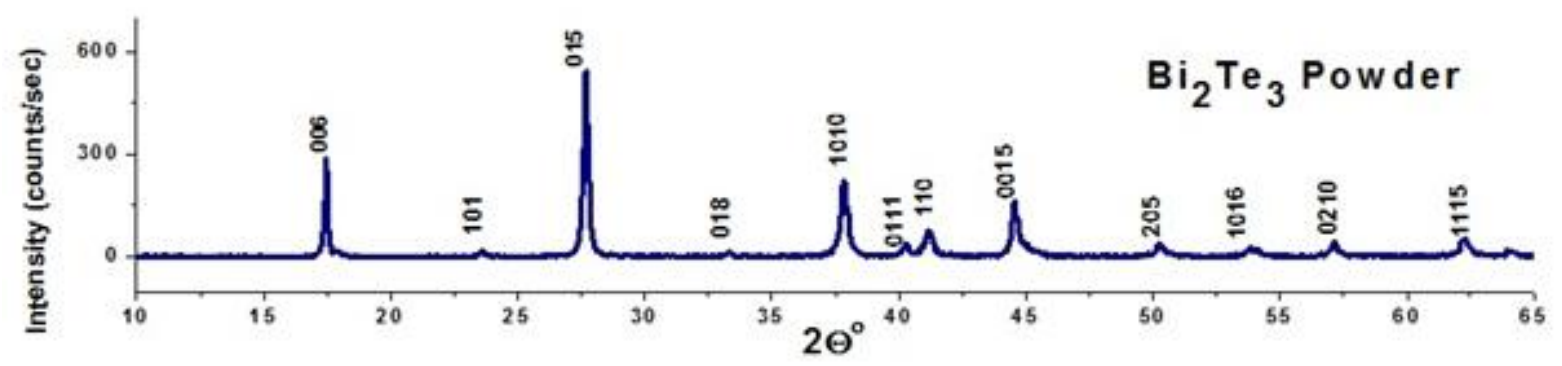

Fig. 1. X-ray diffraction spectra of $\mathrm{Bi}_{2} \mathrm{Te}_{3}$ powder

Table 1 shows the values of Miller indices, ( $\mathrm{k} \mathrm{k}$ ), for highestdiffraction peak together with the interplanar spacing, $\left(\mathrm{d}_{\mathrm{hkl}}\right)$, and our check cell is in agreement with [23-27]. Fig. 2(a) shows the XRD pattern of thermally evaporated $\mathrm{Bi}_{2} \mathrm{Te}_{3}$ thin films of thickness $27 \mathrm{~nm}$ grown at substrate temperature $298 \mathrm{~K}$, Fig. 2(b) shows theXRD pattern of thermally evaporated $\mathrm{Bi}_{2} \mathrm{Te}_{3}$ thin films of thickness $36 \mathrm{~nm}$ grown at substrate temperature $623 \mathrm{~K}$. The diffraction pattern exhibits a broad peak around $2 \theta=27^{\circ}$, indicating that the evaporated $\mathrm{Bi}_{2} \mathrm{Te}_{3}$ film is amorphous. Fig. 2(c,d)shows the XRD pattern of irradiated films The absence of sharp diffraction peaks and thepresence of humps emphasize amorphous nature.

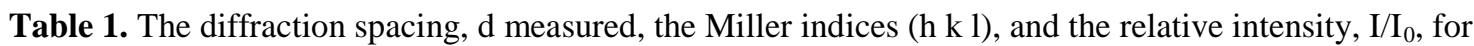
$\mathrm{Bi}_{2} \mathrm{Te}_{3}$ in the powder form

\begin{tabular}{|l|l|l|l|l|l|}
\hline no & $2 \theta$ (degree) measured & $\mathrm{d}\left(\mathrm{A}^{\circ}\right)$ measured & $\mathrm{d}\left(\mathrm{A}^{\circ}\right)$ calculated & I/Io & hkl \\
\hline 1 & 17.46732 & 5.07292 & 5.07305 & 57.60 & 006 \\
\hline 2 & 27.68051 & 3.22002 & 3.22010 & 100.00 & 015 \\
\hline 3 & 37.84259 & 2.37543 & 2.37549 & 38.76 & $101 \underline{0}$ \\
\hline
\end{tabular}




\begin{tabular}{|l|l|l|l|l|l|}
\hline 4 & 41.14973 & 2.19184 & 2.19189 & 14.38 & 110 \\
\hline 5 & 44.54489 & 2.03234 & 2.03239 & 29.19 & $001 \underline{5}$ \\
\hline
\end{tabular}

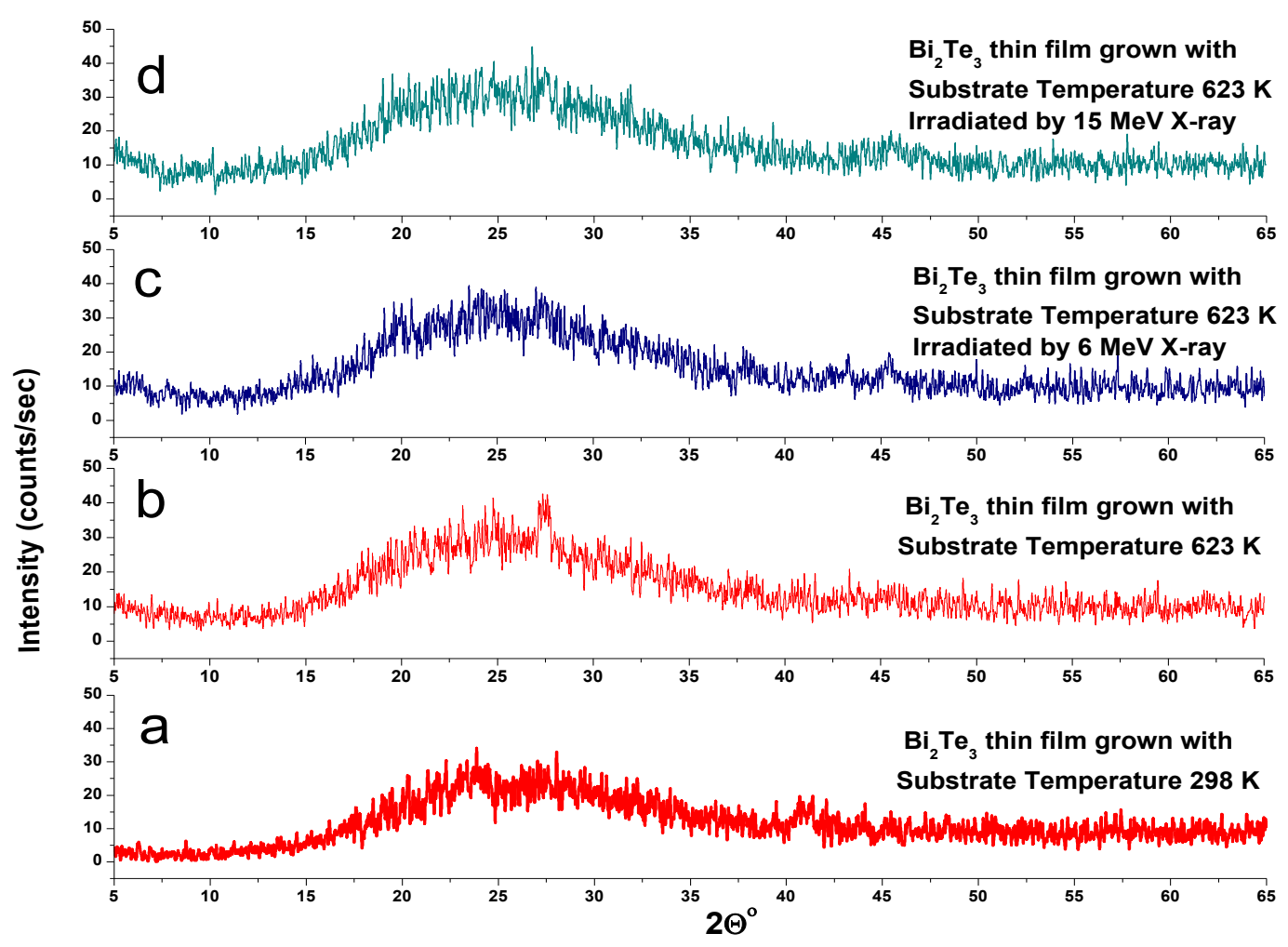

Fig. 2. X-ray diffraction spectra of (a) as-grown thin film at substrate temperature $298 \mathrm{~K}$ and (b) as-grown thin film at substrate temperature $623 \mathrm{~K}$ and (c) irradiated Bi2Te3 thin films with high energy X-ray (6 MeV) and (d) irradiated Bi2Te3 thin films with high energy $\mathrm{X}$-ray $(15 \mathrm{MeV})$.

\subsection{Topological and morphological characterizations}

The surface topography and grain shape growth for as-grown and irradiated $\mathrm{Bi}_{2} \mathrm{Te}_{3}$ thin films are investigated by scanning electron microscopy (SEM) as shown in Figure 3.The topograph (a) for as-grown $\mathrm{Bi}_{2} \mathrm{Te}_{3}$ thin films at substrate temperature $298 \mathrm{~K}$ shows clearly almost uniform of thin film onto glass substrate distribution of granular shape Nano crystallite particles.The topograph (b) for as-grown $\mathrm{Bi}_{2} \mathrm{Te}_{3}$ thin films at substrate temperature $623 \mathrm{~K}$ shows visibly almost uniform of film distribution of granular shape Nano crystallite particles. The topograph (c) shows the surface morphology of the -irradiated film with high energy X-ray $(6 \mathrm{MeV})$, where the particles are observed with higher aggregation and pores appear compared with that of the as-deposited film. The topograph (d) shows the surface morphology of the -irradiated film with high energy X-ray $(15 \mathrm{MeV})$, which shows more pores, in other words, the X-ray radiation can modify the surface topographies of $\mathrm{Bi}_{2} \mathrm{Te}_{3}$ thin films by controlling the aggregate densification and porous properties of $\mathrm{Bi}_{2} \mathrm{Te}_{3}$ thin films[28].

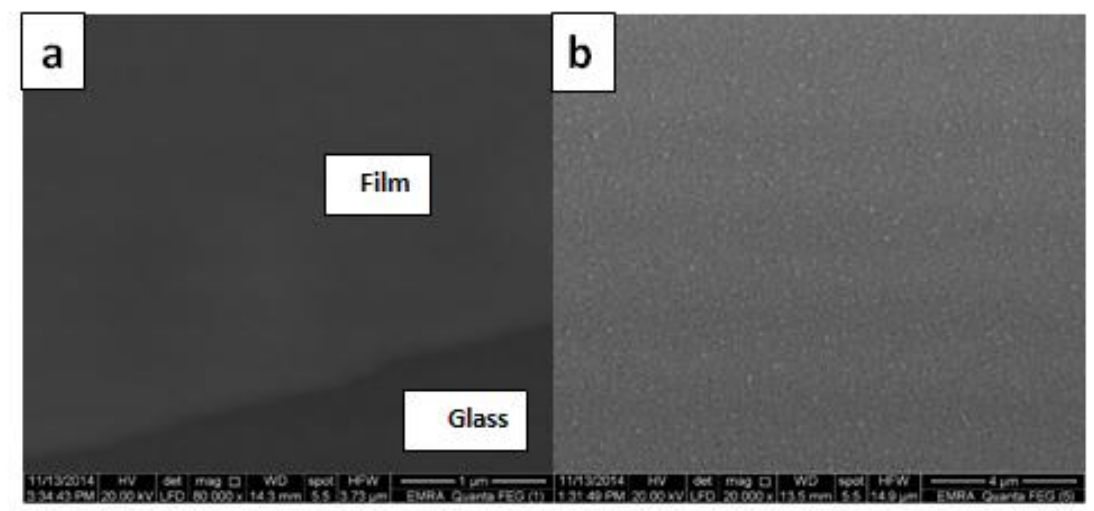




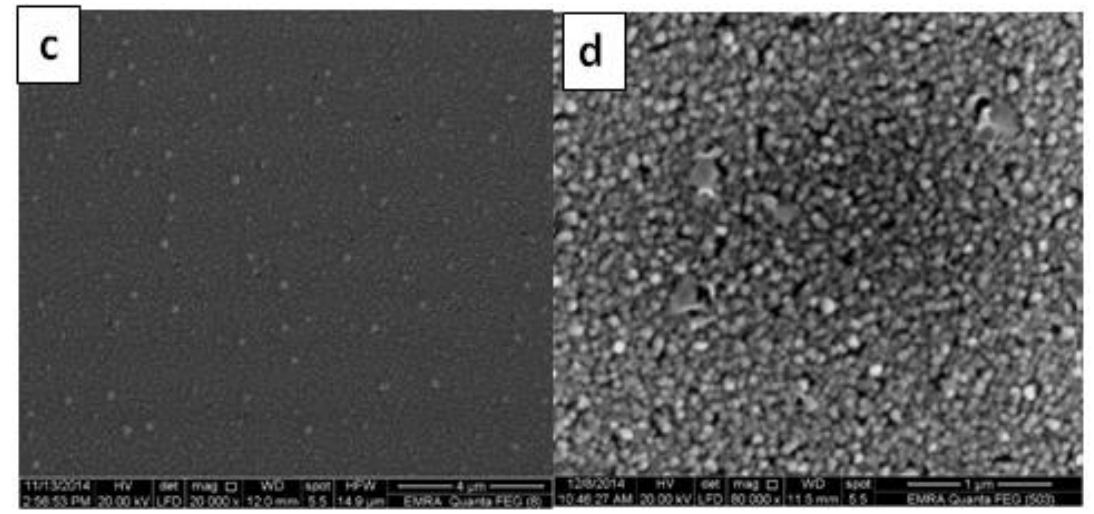

Fig. 3. SEM images (a) as-grown thin film at substrate temperature $298 \mathrm{~K}$ and (b) as-grown thin film at substrate temperature $623 \mathrm{~K}$ and (c) irradiated $\mathrm{Bi}_{2} \mathrm{Te}_{3}$ thin films with high energy X-ray $(6 \mathrm{MeV})$ and (d) irradiated $\mathrm{Bi}_{2} \mathrm{Te}_{3}$ thin films with high energy X-ray $(15 \mathrm{MeV})$.

\subsection{Optical characterizations}

\subsubsection{Transmission and reflection spectra}

Fig.4 (a.b) shows Spectral distribution of $\mathrm{T}(\lambda)$ and $\mathrm{R}(\lambda)$ of as-grown and irradiatedBi $\mathrm{Te}_{3}$ thin films. The spectral distribution shows two abrupt changes at the first of UV region $(\lambda=260 \mathrm{~nm})$ and the first of IR region $(\lambda=810 \mathrm{~nm})$ which represent an optical switch behavior at $\lambda=260$ and $\lambda=810$. All-optical switching mechanism based on the optical control of resonance energy transfer (RET) between particles When RET occurs spontaneously, following the absorption of light, it usually represents the principal process for the intermolecular translation of electronic energy, from the sites of initial optical excitation [29].However, under suitable conditions such processes of energy transfer can be activated or deactivated by (non-resonant) optical stimulation. From the Figure it is evident that the optical spectral distribution is sensitive to the radiation influence.Reflectance intensity decreaseat UV region; wavelength range from 190 to $260 \mathrm{~nm}$ and IR region; wavelength range from 810 to $2500 \mathrm{~nm}$.
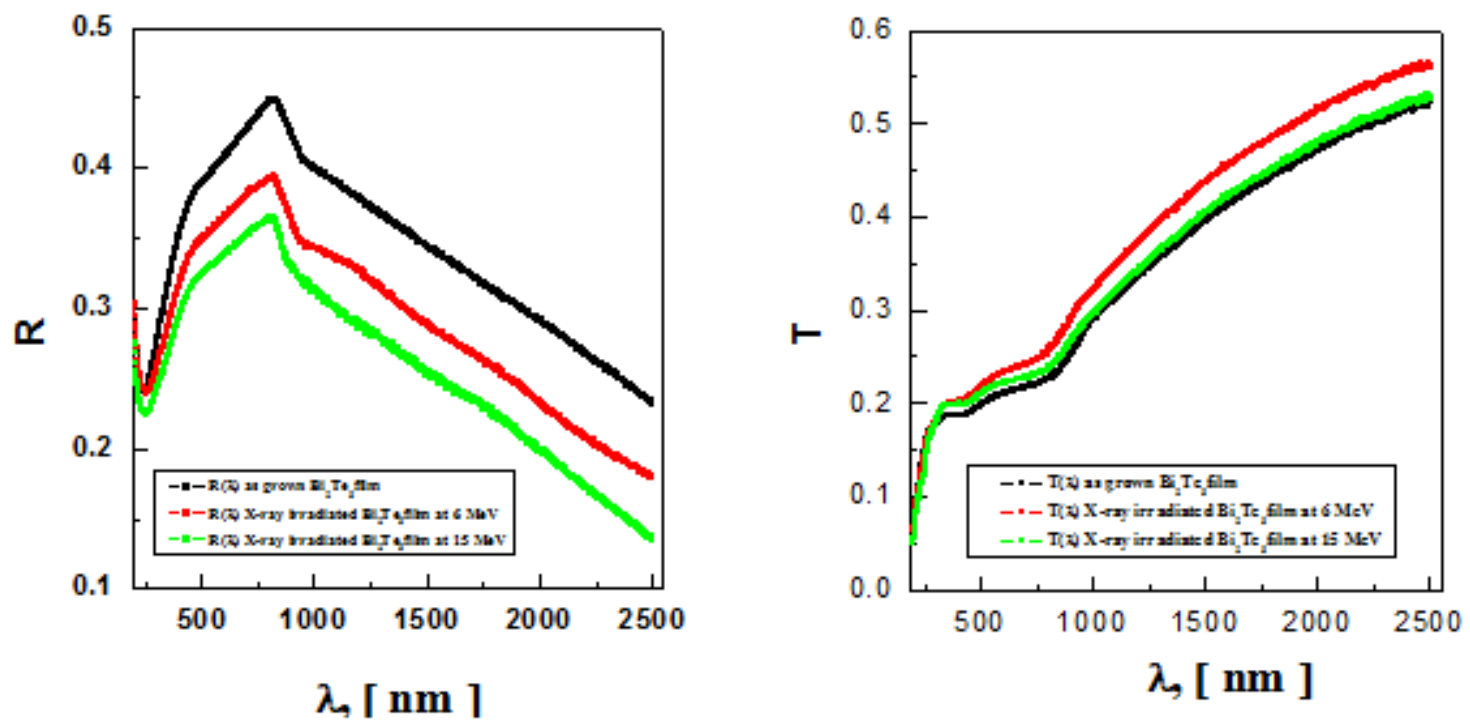

Fig. 4. Spectral distribution of $\mathrm{T}(\lambda)$ and $\mathrm{R}(\lambda)$ of as-grown and irradiated $\mathrm{Bi}_{2} \mathrm{Te}_{3}$ thin film

\subsubsection{Optical dispersion characteristics}

Real part of the indices, $\mathrm{n}(\mathrm{h} v)$, and imaginary part, $\mathrm{k}(\mathrm{hv})$, of refraction for as-grown and irradiated $\mathrm{Bi}_{2} \mathrm{Te}_{3}$ thin films calculated from eq (2) and (3) showed in Fig 5 and 6 as a function of photon energy. The values of the real part of the indices of refraction exhibits an anomalous dispersion in the range $\lambda<850 \mathrm{~nm}$, which can be explained according to the multi-oscillator model [30]. The refractive index for all films exhibits a normal dispersion in the range $\lambda>1000 \mathrm{~nm}$, which can be understood by using the single oscillator model [31,32]. The results for the real part, $\mathrm{n}$, show one peak at $815 \mathrm{~nm}$ in the first of IR. The intensity ofthis peakis descending, i.e., decrease with increasing the energy of $\mathrm{X}$-ray radiation. At longer wavelength $(\mathrm{h} v \rightarrow 0)$ the calculated value of the refractive index decreases by an amount $\Delta \mathrm{n}=0.31$ and 0.54 after irradiation process for 6 $\mathrm{MeV}$ and $15 \mathrm{MeV}$, respectively.The decrease in the refractive index values is attributed to the decrease of mass 
density [33].Optical absorption analysis has widely proven to be an important and efficient tool in exploring and interpreting the various phenomena of electronic structures and processes in the materials subjected to radiation [34-25].

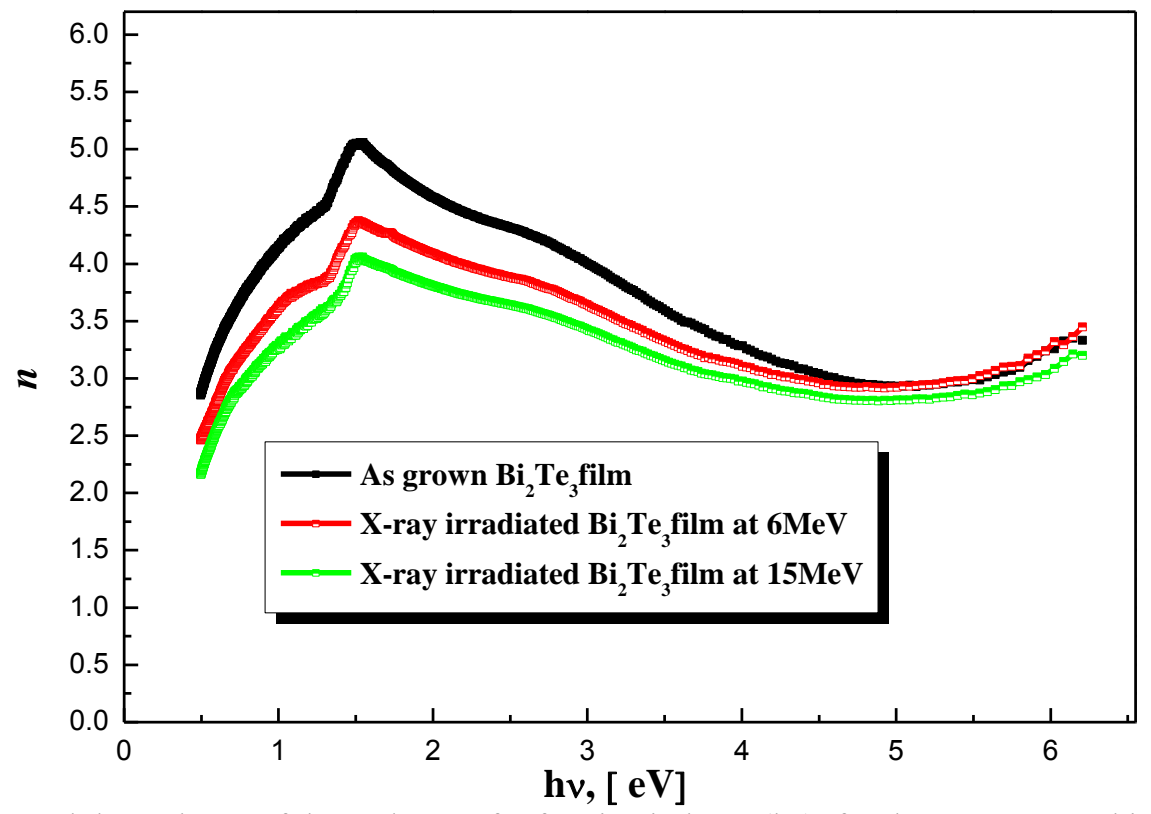

Fig. 5. The spectral dependence of the real part of refractive index, $n(h v)$, for the as grown and irradiated $\mathrm{Bi}_{2} \mathrm{Te}_{3}$ thin films .

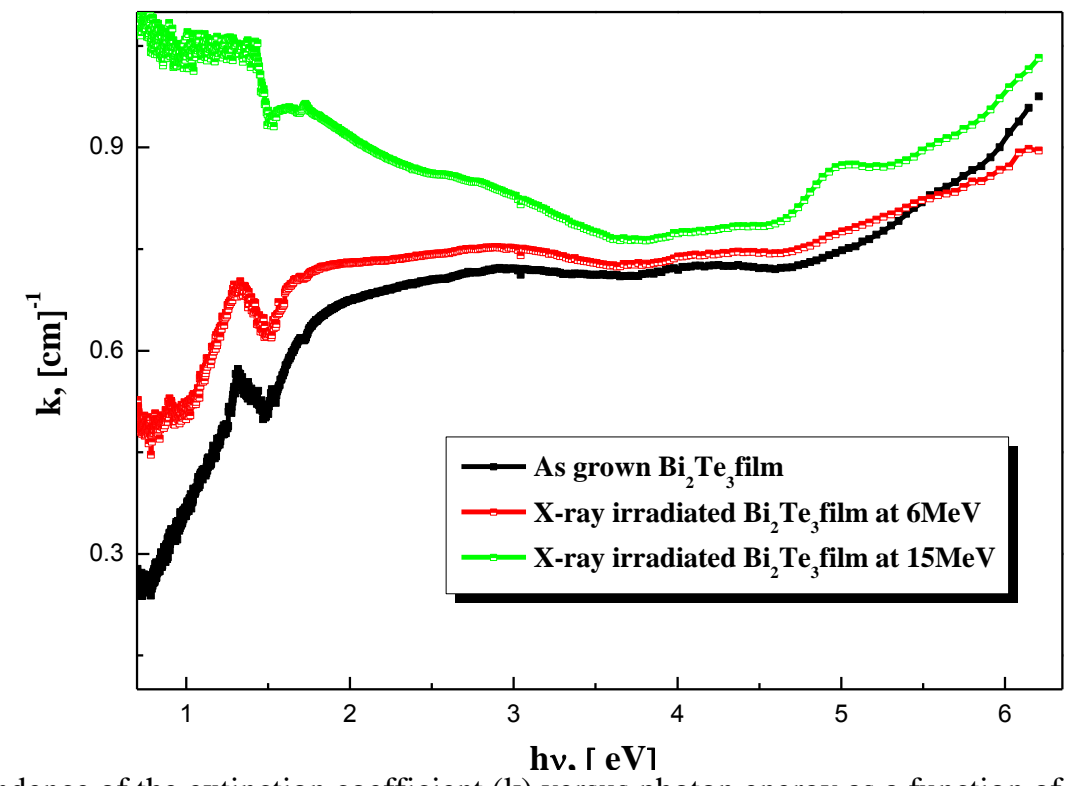

Fig. 6. The dependence of the extinction coefficient (k) versus photon energy as a function of irradiation dose.

Fig. 7 shows the variation of the absorption coefficient $(\alpha)$ of as-grown and irradiated $\mathrm{Bi}_{2} \mathrm{Te}_{3}$ thin films with thickness $36 \mathrm{~nm}$, as a function of photon energy, (hv). It is evident that the optical absorption spectral distribution is sensitive to X-ray radiation; a considerable shift in the absorption was observed. The absorption increase for X-ray-irradiated $\mathrm{Bi}_{2} \mathrm{Te}_{3}$ film. At lower optical frequencies, Wemple and DiDomenico $[37,38]$ introduced two parameters based on the single effective oscillatormodel; $\mathrm{E}_{\mathrm{d}}$ to describe the dispersion of the refractive index and thesingle oscillator energy $E_{o}$ to give quantitative information on theoverall band structure of the material"average gap" [39]. The refractive index is expressed in terms of these parameters as in references[37,38]:

$\left(n^{2}-1\right)^{-1}=\frac{E_{o}}{E_{d}}-\frac{1}{E_{o} E_{d}}(h v)^{2}$

Fig. 8 shows the plot of $\left(\mathrm{n}^{2}-1\right)^{-1}$ against $(\mathrm{hv})^{2}$ for as-grown and irradiated $\mathrm{Bi}_{2} \mathrm{Te}_{3}$ thin films. $\mathrm{E}_{\mathrm{o}}$ and $\mathrm{E}_{\mathrm{d}}$ can be obtained from the slope and intercept of the fitted straight lines of the experimental points. The infinite 
wavelength dielectric constant $(\varepsilon \infty)$ can be deduced from extrapolation the linear part of the optical dispersion at zero photon energy $\mathrm{n}(0)$. The results for as-grown and irradiated $\mathrm{Bi}_{2} \mathrm{Te}_{3}$ thin films are calculated in Table 2 .

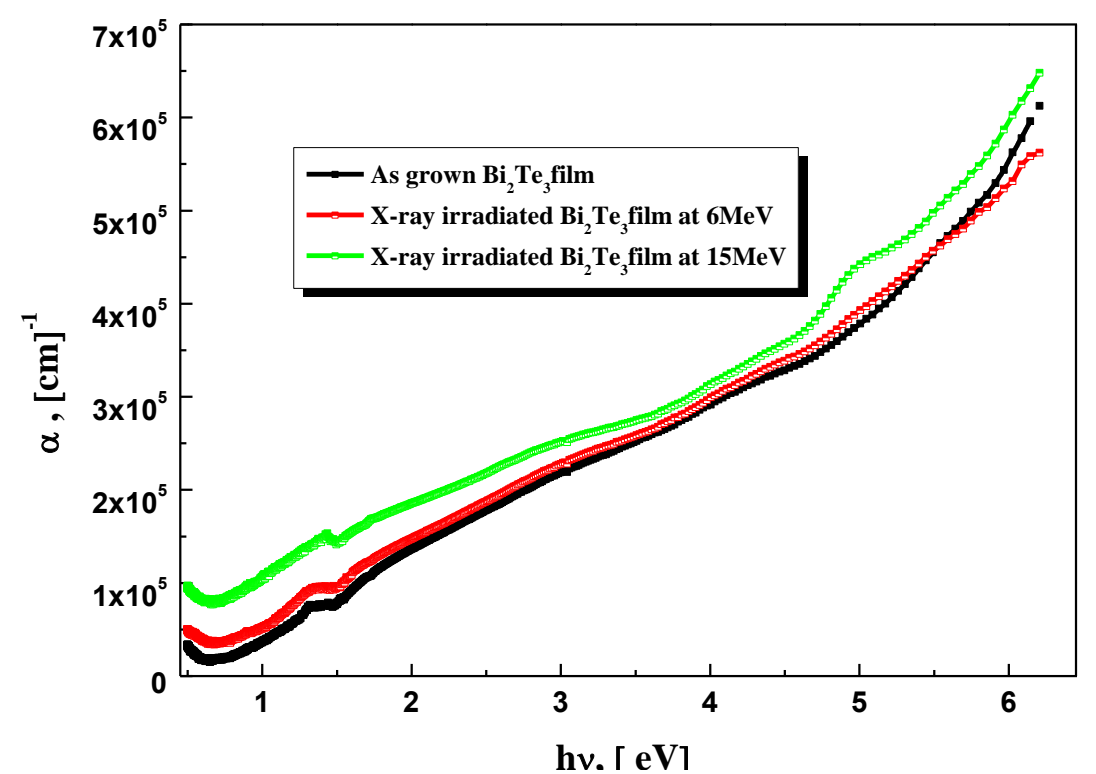

Fig. 7. The dependence of the absorption coefficient $(\alpha)$ on Photon energy as a function of irradiation dose.

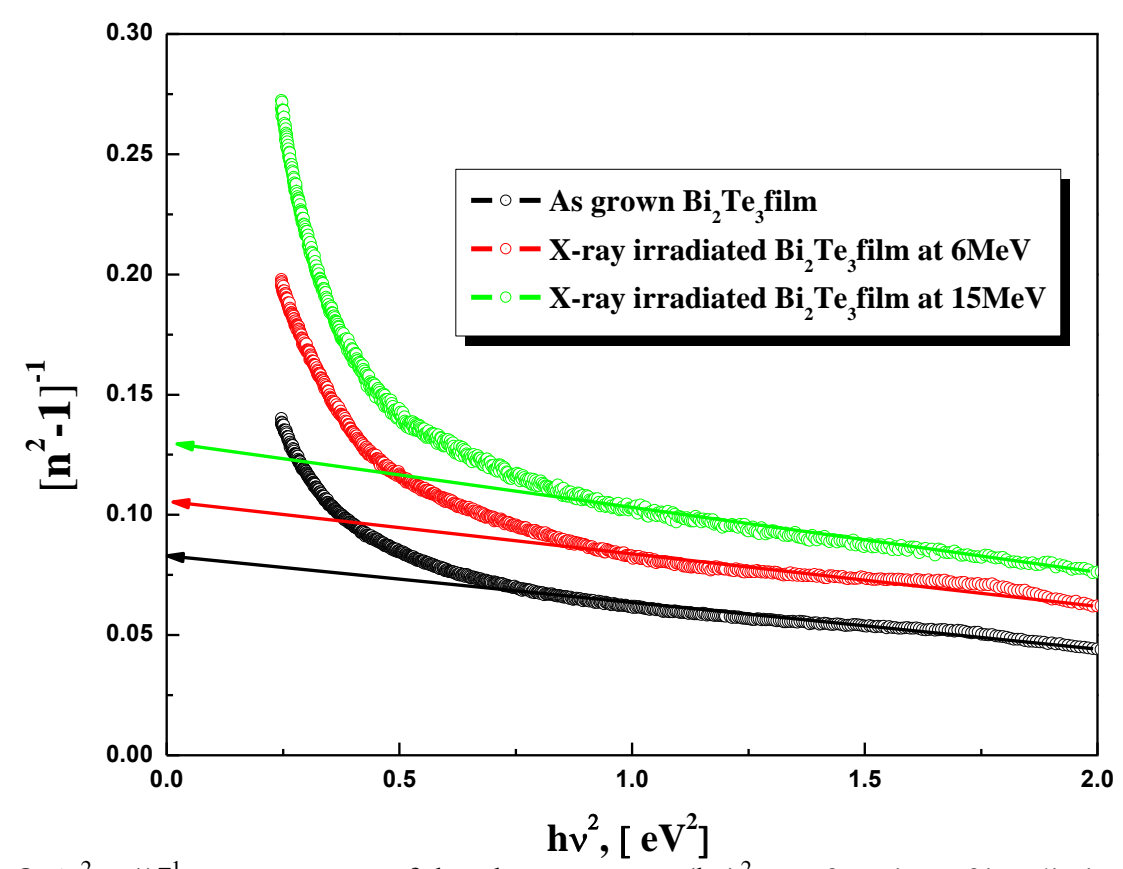

Fig. 8. $\left(n^{2}-1\right)^{-1}$ versus square of the photon energy $(h v)^{2}$ as a function of irradiation dose

The relationship between the lattice dielectric constant $\left(\varepsilon_{\mathrm{L}}\right)$ and refractive index, $\mathrm{n}$, is given by [32]:

$n^{2}=\varepsilon_{l}-\left(\frac{e^{2}}{4 \pi^{2} \varepsilon_{o} C^{2}}\right)\left(\frac{N}{m^{*}}\right) \lambda^{2}(6)$

Where $\varepsilon_{\mathrm{L}}$ is the high frequency dielectric constant, e is the elementary charge, $\varepsilon_{\mathrm{o}}$ is the permittivity of free space and $\left(\mathrm{N} / \mathrm{m}^{*}\right)$ is the ratio of free carrier concentration to the free carrier effective mass. Fig. 9 shows the linear relation between $\mathrm{n}^{2}$ and $\lambda^{2}$ for the as-grown and irradiated $\mathrm{Bi}_{2} \mathrm{Te}_{3}$ thin films. Extrapolating the linear parts to zero wavelengths indicated the value 11.22 for $\varepsilon_{\mathrm{L}}$ of the as-grown film, andthe values 12.95 and 17.12 for $\varepsilon_{\mathrm{L}}$ of the X-ray irradiated $\mathrm{Bi}_{2} \mathrm{Te}_{3}$ thinfilms by energies $6 \mathrm{MeV}$ and $15 \mathrm{MeV}$, respectively. Theratio of free carrier concentration could be calculated from the slopeof these linear parts, where the ratio $\left(\mathrm{N} / \mathrm{m}^{*}\right)$ was considered tobe $4.24 \times 10^{44} \mathrm{Kg}^{-1} \mathrm{~m}^{-1}$ for the as-grown and $4.54 \times 10^{44} \mathrm{Kg}^{-1} \mathrm{~m}^{-1}, 4.75 \times 10^{44} \mathrm{Kg}^{-1} \mathrm{~m}^{-1}$ for X-ray 
irradiated $\mathrm{Bi}_{2} \mathrm{Te}_{3}$ thin films by energies $6 \mathrm{MeV}$ and $15 \mathrm{MeV}$, respectively. The disagreement between $\varepsilon_{\infty}$ and $\varepsilon_{\mathrm{L}}$ may be due to the differences in free carrier contribution [40].

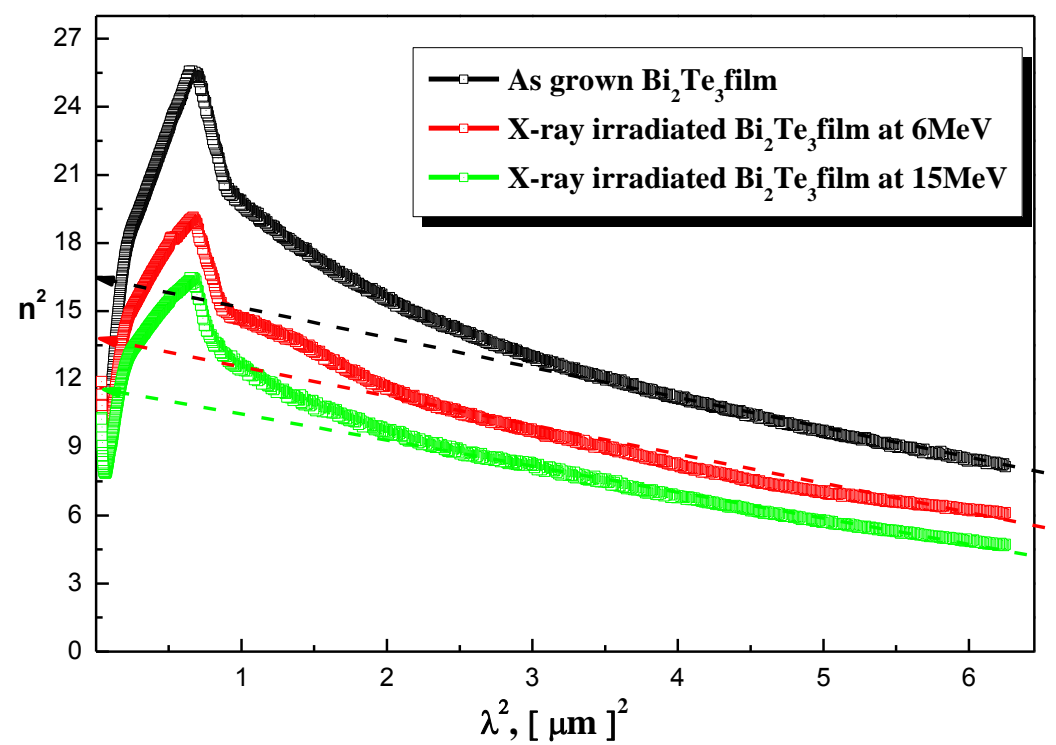

Fig. 9. $n^{2}$ versus square of the wavelength $\left(\lambda^{2}\right)$ as a function of irradiation dose.

\subsubsection{Energy gap determination}

The band structure and the existence of an energy gap are believed to be dependent upon the arrangement of nearest atomic neighbors and the existence of local or short-range order. Mott was the first who proposed the concept of short-range order in amorphous solids [41].The types of transition and the value of optical energy gap can be demonstrated by Bardeen et al. [42] as

$\alpha h v=\alpha_{o}\left(h v-E_{g}\right)^{r}$

Fig. 10shows the plots of $(\alpha h v)^{1 / 2}$ versus (hv) for as-grown and irradiated $\mathrm{Bi}_{2} \mathrm{Te}_{3}$ thin films. The indirect band gaps $\mathrm{E}_{\mathrm{g}}$ onset for the as-grown and irradiated films are evaluated from the $\mathrm{x}$-axis intercepts at $(\alpha \mathrm{hv})^{1 / 2}=0$. According to Eq.(7), the values of the corresponding energies listed in table 2. These energies were found to be $0.35 \mathrm{eV}$ for the as-grown and 0.22 and $0.05 \mathrm{eV}$ for the irradiated films by $6 \mathrm{MeV}$ and $15 \mathrm{MeV}$, respectively. The amorphous nature of our sample may be confirmed by the indirect transition rather than direct transition [43]. The variation of the optical energy gap with electron irradiation energies and doses can be explained as the change in the degree of disorder. From the density-of-state model, it is known that $\mathrm{E}_{\text {opt }}$ decreases with an increasing degree of disorder of the amorphous phase [44].

Table.2. Effect of exposure times on the optical parameters $(\mathrm{Eg}),(\mathrm{Eo}),(\mathrm{Ed}),(\varepsilon \infty),(\varepsilon \mathrm{L})\left(\mathrm{N} / \mathrm{m}^{*}\right)$ and $\mathrm{Nc}$.

\begin{tabular}{|c|c|c|c|c|c|c|c|}
\hline X-ray Energy & $E_{g}(e V)$ & $E_{0}(e V)$ & $E_{d}(e V)$ & $\square_{\square}$ & 9 & $\mathbf{N} / \mathbf{m}^{*}\left(\mathrm{Kg}^{-1} \mathbf{m}^{-1}\right)$ & $\mathrm{Nc}$ \\
\hline As-grown & 0.35 & 2.19 & 28.49 & 13.9 & 11.22 & $4.24 \times 10^{44}$ & 4.14 \\
\hline $6 \mathrm{MeV}$ & 0.22 & 2.36 & 23.57 & 11.0 & 12.95 & $4.54 \times 10^{44}$ & 3.43 \\
\hline $15 \mathrm{MeV}$ & 0.05 & 2.13 & 16.22 & 8.63 & 17.12 & $5.75 \times 10^{44}$ & 2.36 \\
\hline
\end{tabular}

An important achievement of the WD model is related to the dispersion energy to the other physical parameters of the materials through an empirical formula [45, 46]:

$E_{d}=\beta N_{c} Z_{a} N_{e}$

where $\beta$ is a constant, and equals $0.37 \pm 0.04 \mathrm{eV}$ for covalently bonded crystalline and amorphous chalcogenides, $\mathrm{Nc}$ is the coordination number of the cation that is the nearest neighbor to the anion, $\mathrm{Ne}$ is the effective number of valence electrons per anion and $\mathrm{Za}$ is the formal chemical valence of the anion (here $\mathrm{Za}=2$ for $\mathrm{Bi}_{2} \mathrm{Te}_{3}$ ), $\mathrm{Ne}$ has the value equal to 9.3. The obtained value of coordination number from Eq. (8) listed in Table.2.

Various theoretical models were proposed by various researchers forthe estimation of the third order susceptibility, $\chi^{(3)}$ and nonlinear refractive index, $\mathrm{n}_{2}$ [47]. Ticha' et al. Have combined Miller generalized rule and static refractive index (estimated by WDD single-oscillatormodel) [48-51]. According to that model, 
the following relationswere used for the estimation of the third order susceptibility, $\chi^{(3)}$, andnon-linear refractive index $\left(\mathrm{n}_{2}\right)$ :

$\left(\chi^{(3)}\right)=\frac{A}{4 \pi^{4}}\left(n_{o}^{2}-1\right)^{4}$

Where $\left.\mathrm{A}=1.7 \times 10-10, \chi^{(3)}\right)$ is measured in esu and $\mathrm{n}_{\mathrm{o}}$ is the static refractive index.

$n_{2}=\left(12 \pi\left(\chi^{(3)}\right) / n_{o}\right) \quad(10)$

Irradiation dose dependent third-order susceptibility and non-linear refractive index are tabulated in Table 3.

Table .3. Irradiation dose dependent third-order susceptibility and non-linear refractive index

\begin{tabular}{|l|l|l|}
\hline X-ray Energy & $\square^{(3)} \times 10^{-7}($ esu $)$ & $\mathbf{N}_{2} \times 10^{-6}($ esu $)$ \\
\hline As-grown & 0.94 & 0.75 \\
\hline $6 \mathrm{MeV}$ & 1.71 & 1.26 \\
\hline $15 \mathrm{MeV}$ & 6.11 & 3.87 \\
\hline
\end{tabular}

\section{Summary and conclusions}

The main conclusions can be summarized as follows. The analysis of XRD of $\mathrm{Bi}_{2} \mathrm{Te}_{3}$ proved that the received powder material form polycrystalline patterns with hexagonal structure and the as-grown $\mathrm{Bi}_{2} \mathrm{Te}_{3}$ thin films have amorphous structure. X-ray irradiation affected the measured values of transmission, reflectance and absorption spectra. In the spectral range 200-2500 $\mathrm{nm}$ the refractive index showed anomalous dispersion in the wavelength range of $(\lambda<1000 \mathrm{~nm})$ while in the wavelength more than $1000 \mathrm{~nm}$, it is found that the refractive index dispersion data obeyed the single oscillator model. The advantage of using the single oscillator equation for the fit of the experimental data is that, it provides an indirect rather than direct band gap. The X-ray irradiation has an effect on the excitonic and impurities levels, this may due to the change in the microstructure of the film. The type of electronic transition responsible for optical properties is an indirect allowed transition. On the other hand, the analysis examines the change in the refractive index of the as-deposited and irradiated films. It is found that the refractive index dispersion data obeyed the single oscillator model. An interpretation of single oscillator parameters and Drude mode of free carriers absorption have been described for the analysis of refractive index dispersion before and after irradiation.

[1]. D. A. Wright, Nature, 1958, 181, 834.

\section{References}

[2]. D.M. Rowe, C.M. Bhandari, Modern Thermoelectrics, Reston Publishing Company, Reston, VA, 1983.

[3]. K. Watanabe, N. Sato, S. Miyaoka, New optical recording material for video disc system, Journal of Applied Physics, 54 (1983) 1256-1260.

[4]. ZhenHua Wang, Liang Yang, XiaoTian Zhao, ZhiDong Zhang and Xuan P. A. Gao,Nano research, 2014.

[5]. C. Desai, M. Kotak, D. Vyas, P. Soni, Photoconductivity of Sb0. 2Bi1. 8Te3 thin films, SPIE proceedings series, DOI (2000) 14641467.

[6]. H. Zhang, C. X. Liu, X. L. Qi, X. Dai, Z. Fang and S. C. Zhang, Nat.Phys., 2009, 5, 438.

[7]. Kane, C.L., Mele, E.J.: Quantum spin Hall effect in graphene. Phys. Rev. Lett. 95, 226801 (2005)

[8]. Hasan, M.Z., Kane, C.L.: Colloquium: Topological insulators. Rev. Mod. Phys. 82, 3045 (2010)

[9]. Bychkov, Y.A., Rashba, E.I.: Properties of a 2D electron gas with lifted spectral degeneracy. JETP Lett. 39, 66 (1984)

[10]. HelinZouD.M.RoweGaoMin,Growthofp-andn type bismuth telluride thin films by co-evaporation,J.Cryst.Growth222(2001)82-87.

[11]. A.Mzerd,D.Sayah,J.C.Tedenac,A.Boyer,Optimal crystal growth conditions of thin films ofBi2Te3 semiconductors, J.Cryst. Growth 140(1994)365-369.

[12]. F.Volklein,V.Baier,U.Dillner,E.Kessler,Transport properties of flash-evaporated (Bi1-xSbx) ${ }_{2} \mathrm{Te}_{3}$ films I:optimizationof film properties, ThinSolid Films 187(1990)253-262.

[13]. M.Takahashi,Y.Katou,K.Nagata,S.Furuta,The composition and conductivity of electro deposited Bi-Te alloy films, Thin Solid Films 240 (1994)70-72.

[14]. Y.H.Shing,Y.Chang,A.Mirshafii,L.Hayashi,S.S.Roberts,J.Y.Josefouriez, N. Tran,Sputtered Bi ${ }_{2} \mathrm{Te}_{3}$ and PbTe thin films, J.Vac.Sci. Technol. A1 (2)(1983) 503-506.

[15]. Zhuang-haoZheng,PingFan,Guang-xingLiang,Dong-pingZhang,XingbaoChen,Annealingtemperatureinfluenceonelectricalproper- ties J.Phys.Chem.Solids71(2010) 1713-1716.

[16]. HyunwooYou,Sung-hwanBae,JongmanKim,Jin-sangKim,ChanPark, Deposition of Nanocrystalline Bi2Te3 Films using amodified MOCVD system, J. Electron.Mater.40(2011)635-640.

[17]. YuanDengHui-minLiangYaoWang,Zhei-weiZhang,MingTanJiao-linCui, Growth andtransport properties of oriented bismuth telluride films, J.Alloys Compd. 509(2011)5683-5687.

[18]. R. El Mallawany, A.A. El Rahamani, A. Abousehly, E. Yousef, Materials Chemistry and Physics 52 (1998) 161.

[19]. E. Atanassova, A. Paskaleva, R. Konakova, D. Spassov, V.F. Mitin, Microelectronics Journal 32 (2001) 553.

[20]. K. Arshak, O. Korostynska, Annals of Physics 13 (2004) 87.

[21]. M.M. El-Nahass, Journal of Materials Science 27 (1992) 6592.

[22]. M.M. El-Nahass, A.F. El-Deeb, H.S. Metwally, H.E.A. El-Sayed, A.M. Hassanien, Solid State Sciences 12 (2010) 552

[23]. H Koc, AM Mamedov, E Ozbay - Ferroelectrics, 2013 - Taylor \&Francis, Optical Properties and Electronic Band Structure of Topological Insulators (on A5 2B6 3 Compound Based)

[24]. G. Wang and T. Cagin, Electronic structure of the thermoelectric materials Bi2Te3 and Sb2Te3from first-principles calculations. Phys Rev B. 76, 075201.1-075201.8 (2007).

[25]. R. W. G. Wyckoff, Crystal Structures. New York: Wiley 1964;2:30.

[26]. Bhakti Jariwala, Dimple shah, N.M. Ravindra, Influence of Doping on Structural and Optical Properties of Bi 2Te3 Thin Films Thin Solid Films (2015) 
[27]. S. E. Harrison,1,a) L. J. Collins-McIntyre,2 S. Li,3 A. A. Baker,2,4 L. R. Shelford,4 Y. Huo,1A. Pushp,5 S. S. P. Parkin,5 J. S. Harris,1 E. Arenholz,6 G. van der Laan,4 and T. Hesjedal2,b , Study of Gd-doped Bi2Te3 thin films: Molecular beam epitaxy growth and magnetic properties - JOURNAL OF APPLIED PHYSICS 115, 023904 (2014)

[28]. M.M. El-Nahassa,H.M. Abd El-Khalek, Ahmed M. Nawar, Topological, morphological and optical properties of Gamma irradiated $\mathrm{Ni}$ (II) tetraphenyl porphyrin thin films, Optics Communications 285 (2012) 1872-188

[29]. D. L. Andrews and A. A. Demidov (Eds.), Resonance Energy Transfer, Wiley, Chichester, United Kingdom (1999).

[30]. G.C. Messenger, M.S. Ash, 2nd edition, Van Nostrand Reinhold, NY, 1992, p. 216, chapter 6.

[31]. K.L. Chopra, Thinfilm phenomena, Robert E. Krieger Publishing Company, Florida, 1979.

[32]. O.S. Heavens, Optical Properties of Thin Solid Films, Dover, New York, 1991

[33]. B. Andreas, I. Breunig, D.K. Buse, Chemical Physics and Physical Chemistry 6 (2005) 1

[34]. K.L. Chopra, Thinfilm phenomena, Robert E. Krieger Publishing Company, Florida, 1979.

[35]. O.S. Heavens, Optical Properties of Thin Solid Films, Dover, New York, 1991.

[36]. K. Arshak, O. Korostynska, J. Harris, D. Morris, A. Arshak, E. Jafer, Thin Solid Films 516 (2008) 1493

[37]. S.H. Wemple, M. DiDomenico Jr., Physical Review B: Condensed Matter 3 (1971)1338.

[38]. S.H. Wemple, Physical Review B: Condensed Matter 7 (1973) 3767.

[39]. M.P. Solomon, C. Sénémaud Schmidt, M.D. Khodja, Physical Review B: Condensed Matter 38 (1988) 13263.

[40]. M.M. El-Nahass, A.A. Atta, E.A.A. El-Shazly, A.S. Faidah, A.A. Hendi, Materials Chemistry and Physics 117 (2009) 390

[41]. N. F.Mott, Philosophical Magazine,Vol.19, (1969), 835-852

[42]. K. Arshak, O. Korostynska, Annals of Physics 13 (2004) 87.

[43]. M.M. El-Nahass, A.H. Ammar, A.A. Atta, A.A.M. Farag, E.F.M. El-Zaidia, Influence of X-ray irradiation on the optical properties of CoMTPPthinfilms, Optics Communications 284 (2011) 2259-2263

[44]. D.H. Kim, E. Byon, G.H. Lee, S. Cho, Effect of deposition temperature on the structural and thermoelectric properties of bismuth telluride thin films grown by co-sputtering, Thin Solid Films 510 (2006) 148-153.

[45]. A. Boulouz, A. Giani, F. Pascal-Delannoy, M. Boulouz, A. Foucaran, A. Boyer, Preparation and characterization of MOCVD bismuth telluride thin films, Journal of Crystal Growth 194 (1998) 336-341.

[46]. V. Goyal, D. Teweldebrhan, A.A. Balandin, Mechanically-exfoliated stacks of thin films of Bi2Te3 topological insulators with enhanced thermoelectric performance, Applied Physics Letters 97 (2010) 133117.

[47]. N. Peranio, M. Winkler, D. Bessas, Z. Aabdin, J. König, H. Böttner, R.P. Hermann, O. Eibl, J. Alloys Comp. 521 (2012) $163-173$.

[48]. S.H. Wemple, M.D. DiDomenico, Phys. Rev. B 3 (1971) 1338-1351.

[49]. S.H. Wemple, Phys. Rev. B 7-8 (1971) 3767-3777.

[50]. H. Ticha, L. Tichy, J. Optoelectron. Adv. Mater. 4 (2002) 381-386.

[51]. J. Wyne, Phys. Rev. 178 (1969) 1295-1304. 